\title{
"L'Histoire tragique au service du Prince" Un sens politique de la Trilogie de Des Masures?*
}

\section{VERNET}

L'oubli même ne sauve pas les textes du commentaire. Ignorée de tous, jamais, semble-t-il, réexamînée depuis Faguet et Lebègue, la "Trilogie"1 (ainsi baptisée par Faguet) ne s'avance que déjà nommée et entourée de la rumeur des commentaires que lui ont consacrés ces deux pionniers, l'un en $1883^{2}$ l'autre en $1929 .^{3}$ Et si, pour instituer un autre sens de ce théâtre, il faut mettre au jour le défaut des présentations précédentes, ce ne sera que pour tirer toutes les conséquences que nous imposera l'abandon du savoir canonique, dont l'ombre campe encore, ici et là, dans les creux et les recoins de l'analyse de la dramaturgie du XVIème siècle.

Qu'on se reporte au commentaire subtilement ironique et condescendant de Faguet: il est difficile à l'érudit du siècle dernier d'être homme de goût, car les monuments anciens que l'on déterre ne peuvent apparaître dans le champ de l'esthétique classique que sous les espèces du naîf, du primitif, de l'ébauche; Faguet et Lebègue s'émeuvent des premiers pas de la dramaturgie française et lui pardonnent, attendris, assurant d'une main plus ferme cette enfance dont ils ont déjà lu dans Corneille le prestigieux avenir. "Tu Marcellus eris. ..."

C'est ainsi qu'on peut lire:

Nous retrouvons ensuite la jeune Michol s'entretenant avec elle-même de sentiments incertains encore et qu'elle a peine à démêler. On sait que ces analyses délicates des passions naissantes encore et confuses, sont l'écueil de l'art primitif, le triomphe de l'art achevé. Il me semble que c'est avec un assez grand succès que Desmazures s'est tiré de ce pas. Sa Michol est un peu enfant, mais elle est naturelle et vraie. Une ingénue naturelle, au théâtre, et dans le théâtre du XVIe siècle. C'est chose faite pour attirer l'attention. En l'écoutant, je ne me défends qu'à moitié de songer à la Psyché de Corneille.... 4

* Communication donnée le 28 mai 1980 lors d'une séance de travail de la Societé Canadienne d'Etudes de la Renaissance tenue à l'Université de Montréal.

Les dépouillements des Archives de Meurthe-et-Moselle nécessaires à cet article ont été faites, grâce à une subvention du Queen's Advisory Research Committee, par M. J.-P. André. 
Que faire en un commentaire à moins que l'on ne songe? Voici Lebègue:

Il pense à la Psyché de Corneille; ne peut-on pas évoquer aussi Shakespeare et Musset? (p. 359)

Plus éclectique dans ses goûts, Lebégue n'en est pas moins dans la même ligne critique qui approche le seizième siècle via le dix-septième; toute sa production dramatique est en ce sens pré-classique. On comprendra que cette définition même va apporter et recouvrir un bon nombre de présupposés que nous avons appris avec le temps à dépister et dont nous ne pouvons faire grief à Faguet et Lebègue, car qui, sans eux, parlerait de Des Masures?

Reprenons cependant: il semble que quelque chose pourtant aurait dû avertir nos critiques. Devant l'impossibilité de classer ces oeuvres et l'embarras de ceux qui le précèdent, Lebègue s'écrie,

il est encore plus difficile de classer et d'étiqueter les trois David que le Christus Xylonicus et l'A braham sacrifiant; sont-ce des tragédies? pensez à leurs actions entrecroisées! Sont-ce des mystères? remarquez leur conformité aux règles des unités d'action et de temps, et leur richesse psychologique! L'oeuvre est inexplicable, si l'on n'y cherche pas la trace des événements politiques et religieux auxquels l'auteur a été mêlé, l'influence de l'humanisme qu'il avait si longtemps pratiqué et qu'il n'a jamais complètement abandonné, et celle de la poétique protestante qui allait inspirer la plupart de ses derniers ouvrages. ${ }^{5}$

- croyant ainsi, en bon positiviste, qu'il suffira d'ajouter des "faits" (les événements politiques et religieux, les influences subies par Des Masures) pour que l'explication soit complète. Et de fait, si l'on accepte tous les environs conceptuels de cette méthode, on ne pourra attribuer cette impression tenace d'inachèvement qui refuse d'abandonner le critique "Après lecture, la Trilogie laisse le souvenir d'un ouvrage rempli de longueurs, médiocrement écrit, et parsemé de fines indications psychologiques: Des Masures était mieux doué pour le théâtre de moeurs que pour la tragédie" 6 qu'aux imperfections de l'auteur lui-même ou aux fatalités du développement historique:

Cette tentative hybride méritait un sort meilleur: au milieu de nos froides tragédies imitées de Sénèque elle apportait un peu d'originalité et de naturel; mais en France le classicisme allait triompher: si par son enseignement et ses allusions la trilogie s'accordait avec l'époque des guerres de religion, par sa forme elle eût mieux convenu à celle de Hardy. ${ }^{?}$

J'ajoute immédiatement qu'avec toute la sympathie que je peux avoir pour l'auteur qui me permet d'avoir quelque chose à dire ici, ma conversion esthétique n'est pas encore faite: je trouve la lecture (troisième ou 
quatrième) de la trilogie bien longue! Pas encore dis-je: le but de cette étude est d'en placer les conditions de possibilité.

Revenons donc à notre berger. ${ }^{8}$

Le David de Des Masures tiendrait ses origines dans l'appartenance de l'auteur au protestantisme bien sûr, puisqu'une longue tradition en fait effectivement l'exemplum privilégié par les protestants de l'élu du Seigneur, espoir et préfiguration du peuple élu que sont maintenant les sectateurs du vrai Dieu. Mais, comme il convient à un théâtre primitif, la "psychologie," dont Lebègue fait grand cas, vient de l'identification de l'auteur à son protagoniste, et les personnages secondaires de l'observation des personnes qui ont été mêlées à la vie de l'auteur:

Mais il est une oeuvre qui évoque sous un déguisement biblique l'affaire de Saint-Nicolas, c'est la Trilogie. Si la première pièce n'a pour but que d'encourager les protestants à persévérer dans leur lutte, les deux suivantes contiennent des allusions qui ont échappé aux critiques modernes, mais qui étaient claires pour les Lorrains contemporains de l'auteur: en particulier, le personnage de Doëg, ce courtisan traître qui joue dans David Triomphant et David Fugitif un rôle bien plus important que dans la Bible, a été composé d'après le bailli de Nancy et d'autres conseillers du duc. $^{9}$

C'est ainsi qu'à l'aide d'une sorte de bon sens esthétique Des Masures produit des personnages "naturel[s] et vrai[s]" (Faguet à propos de Michol), ${ }^{10}$ qui sont par conséquent "naturel[s] et touchant[s]" six lignes plus loin.

Par l'intermédiaire de l'identification auteur-personnage appuyée sur la biographie, on peut maintenant atteindre une intention, qui s'avère morale (édifiante) donc hors du champ de la critique: "Ainsi donc, dans les trois pièces, tout est subordonné à une intention édifiante; aussi, avant de procéder à une étude littéraire, convient-il de voir comment elle a été réalisée." 11

Cette morale étant tirée des Ecritures, avec la rigueur que l'on connaît aux calvinistes, il en faut aussi conclure que cette édification "a un but" aussi religieux; mais nous sommes en période de guerres de religion, le but est donc aussi bien politique:

Choix du sujet, peinture de certains caractères, réflexions et prêches remplissant les prologues et épilogues et disséminés dans les pièces, tels sont les moyens par lesquels l'auteur a atteint son double but: répandre la foi calviniste et prévenir contre les dangers de la Cour. ${ }^{12}$

Passons sur la critique de l'intentionnalité: nous avons changé tout cela. Remarquons seulement que morale, politique et religion sont les scories de l'analyse, de cet épurement du donné textuel exactement parallèle à 
l'épurement de la dramaturgie française au cours du dix-septième siècle que la critique classique donne à lire dans son histoire littéraire. Si le théâtre du seizième siècle en France souffre esthétiquement de ne pas être encore celui du dix-septième, il faut bien comprendre que celui du dix-septième resplendit de tous les défauts de son prédécesseur qu'il n'a pas: une absence le nimbe. On n'en sortira pas: celui-ci sera toujours, et en même temps, trop et pas assez.

De fait, il ne sera pas surprenant que l'ensemble de l'analyse se laisse assez bien systématiser, et selon une grille que nous reconnaîtrons et que nous prendrons dans la situation suivante:

Malgré ses longueurs et ses maladresses, David triomphant est un des drames psychologiques les plus curieux du XVIème siècle. En effet, Des Masures a tiré du texte biblique une comédie politique... Cet art de filer la scène, de motiver les actes par les réactions sentimentales des personnages, de mettre au premier plan le drame intérieur de David, bref cette prédominance de la psychologie dans l'action ne provient certainement pas des mystères! On peut l'attribuer à l'expérience qu'avait l'auteur des intrigues de Cour, à la lecture de l'A braham sacrifiant, et surtout à celle des tragiques, des comiques anciens, et aussi de Virgile. ${ }^{13}$

D'où nous pouvons extraire:

1. l'auteur se projette dans son oeuvre.

2. la psychologie permet l'accès à la politique. C'est en observant la nature humaine - aidé par les Anciens - que l'on peut expliquer les actes, entre autres l'acte politique. De l'acte aux motifs, et retour, aucune perte.

3. Cette proposition qui est valable pour expliquer la genèse de l'oeuvre l'est aussi pour le lecteur critique contemporain. La politique est accessible à travers la motivation de l'auteur: de ce qu'il condamne la politique, on peut déduire qu'il cherche à valoriser la vie selon Dieu.

4. De même, entre le spectateur ou le lecteur d'alors et nous, peu importent les différences: le sens est tout aussi accessible. En fait, il ne s'agit, à l'aide de faits, que de retrouver ce sens.

Dans ces quatre propositions, rien ne parle que le Sujet.

Or s'agissant de tragédie, et de tragédie protestante, plus de trente ans après le traité $D u$ Serf Arbitre, il faut montrer quelque prudence.

$$
\text { * * * }
$$

On peut partir de l'idée de propagande. Référence: si les tragédies des protestants sont si ennuyeuses, c'est qu'elles prêchent etc. . (voir plus haut). A qui? Au public bien sûr. La réflexion s'est jusqu'à maintenant arrêtée ici, alors que c'est ici que commencent les problèmes. Qui ça, le public? 
Convenons tout de suite que les données manquent; nous n'avons aucune chance d'assigner un sens en produisant une sociologie de la réception. Mais nous savons quand même un certain nombre de choses.

D'abord que les pièces de théâtre n'étaient qu'exceptionnellement jouées plus d'une fois. Que si nous pensons à la diffusion par l'écriture, il faut au moins tenir compte du taux d'alphabétisation extrêmement bas de la population, qui de plus n'est pas en majorité francophone, même si alphabétisation et progrès de la Réforme vont de pair. ${ }^{14}$ En fin nous sommes en 1560 environ, et le partage que prouvent les statistiques plus tardives n'a pas encore dû se faire. Jamais il ne pourrait se trouver assez de lecteurs pour que ceux-ci aient un "poids" politique à notre mode d'aujourd'hui.

Que l'ensemble des auteurs du XVIe siècle, d'accord avec ceux qui dans le domaine politique s'exercent à penser les problèmes de la souveraineté, méprisent totalement tout ceux qui de près ou de loin ne comptent que parce que ça se compte justement; tout ce qui est de l'ordre du quantitatif, le peuple entre autres.

Qu'enfin un protestant n'a que peu de foi en la possibilité pour tout un chacun de pouvoir parvenir à la vérité, en rupture justement avec l'humanisme dont les espoirs sont devenus les axiomes de critiques que nous examinons.

Comprendre, comme tout le reste, est affaire de grâce, gratuite. A ce compte, le théâtre ou le livre ne pourrait prêcher que les convaincus.

$$
\text { * * * }
$$

Qu'est-ce alors que la re-présentation de la Vérité? Quel but peut-elle avoir, et si l'action est à caractère politique, comment la comprendre? On peut suggérer qu'elle ne nous est accessible qu'à condition de perdre le concept de littérature qui n'existait probablement pas de toute façon. Ou du moins qui est inacceptable ici dans le partage qu'il instaure entre le religieux, le politique et le littéraire. Que donc l'oeuvre se replie vers la prière et vers l'acte. C'est tout un. Voyons comment.

$$
\text { * * * }
$$

L'érudition - il en faut - vient ici à notre secours. Il faut rétablir la date de composition doublant ainsi le mouvement de Lebègue, qui a raison de dire que la pièce était écrite en 1563, mais tort probablement de soutenir qu'elle a été publiée pour la première fois à cette date puisque jusqu'à la mort de Calvin, le théâtre - sauf l'Abraham de Bèze - n'a pas droit de cité à Genève. Un peu d'histoire.

Des Masures était au service de la luthérienne Christine de Danemark, duchesse de Lorraine, mère de Charles III de Lorraine, et, au moment qui nous occupe, veuve depuis 1545 de François, duc de Lorraine.

Charles III avait cependant - ou en conséquence, selon qu'on envisage 
les liens très étroits de la maison de Lorraine avec les Guise et la maison royale de France ou l'appartenance de Christine au luthéranisme et à la maison d'Espagne - été littéralement enlevé en avril 1552 par Henri II et élevé à la cour de France parmi les enfants royaux, sans distinction. Il avait donc partagé son enfance avec le Dauphin, le futur François II et ses soeurs Elizabeth, née en 1545 et Claude, née en 1547, celle à qui Grévin dédiera son théâtre en 1561 .

La paix de Cateau-Cambrésis a été signée entre l'Angleterre et la France d'une part en entre l'Espagne et la France d'autre part les 2 et 3 avril 1559, Calais ayant capitulé le 6 janvier. Christine de Danemark a présidé aux négociations, comme médiatrice entre les diverses couronnes. Le même mois sont mariés Claude de France et Charles III de Lorraine. Le traité prévoyait que Philippe II, qui avait essayé ou essayait toujours d'épouser Elizabeth d'Angleterre, épouserait Elizabeth, la fille aînée d'Henri II. Le mariage eut lieu le 22 juin. Le même traité prévoyait aussi que Marguerite, la soeur du roi de France, épouserait Philibert de Savoie, ce qui nous intéresse moins. Enfin, on sait que ce fut lors du tournoi organisé pour célébrer les mariages et la paix qu'Henri II courut une lance de trop, et mourut des suites d'une blessure à l'oeil que lui infligea un éclat de la lance de son chef des gardes protestant Montgomery. Tous les protestants y virent le doigt de Dieu en punition du renouveau de persécutions qui suivit la paix de Cateau-Cambrésis. Anne du Bourg paiera de sa vie, probablement, cette mort accidentelle; la majorité des catholiques cria à la conspiration protestante. C'est ce que nous apprend l'histoire de France.

Voici ce que disait la Bible:

1 David avait achevé de parler à Saül. Et dès lors l'âme de Jonathan fut attachée à l'âme de David, et Jonathan l'aima comme son âme.

2 Ce même jour Saül retint David, et ne le laissa pas retourner dans la maison de son père.

3 Jonathan fit alliance avec David, parce qu'il l'aimait comme son âme.

4 Il ôta le manteau qu'il portait, pour le donner à David; et il lui donna ses vêtements, même son épée, son arc et sa ceinture.

5 David allait et réussissait partout où l'envoyait Saül; il fut mis par Saül à la tête des gens de guerre, et il plaisait à tout le peuple, même aux serviteurs de Saül.

6 Comme ils revenaient, lors du retour de David après qu'il eut tué le Philistin, les femmes sortirent de toutes les villes d'Israël au-devant du roi Saül, en chantant et en dansant, au son des tambourins et des triangles, et en poussant des cris de joie.

7 Les femmes qui chantaient se répondaient les unes aux autres, et disaient:

Saül a frappé ses mille,

Et David ses dix mille. 
8 Saül fut très irrité, et cela lui déplut. Il dit: On en donne dix mille à David, et c'est à moi que l'on donne les mille! Il ne lui manque que la royauté.

9 Et Saül regarda David d'un mauvais oeil, à partir de ce jour et dans la suite.

10 Le lendemain, le mauvais esprit de Dieu saisit Saül, qui eut des transports au milieu de la maison. David jouait, comme les autres jours, et Saül avait sa lance à la main.

11 Saül leva sa lance, disant en lui-même: Je frapperai David contre la paroi. Mais David se détourna de lui deux fois.

12 Saül craignait la présence de David, parce que l'Eternel était avec David et s'était retiré de lui.

13 Il l'éloigna de sa personne, et il l'établit chef de mille hommes. David sortait et rentrait à la tête du peuple;

14 Il réussissait dans toutes ses entreprises, et l'Eternel était avec lui.

15 Saül, voyant qu'il réussissait toujours, avait peur de lui;

16 Mais tout Israël et Juda aimaient David, parce qu'il sortait et rentrait à leur tête.

17 Saül dit à David: Voici, je te donnerai pour femme ma fille aînée Mérab; sers-moi seulement avec vaillance, et soutiens les guerres de l'Eternel. Or Saül se disait: Je ne veux pas mettre la main sur lui, mais que la main des Philistins soit sur lui.

18 David répondit à Saül: Qui suis-je, et qu'est-ce que ma vie, qu'est-ce que la famille de mon père en Israël, pour que je devienne le gendre du roi?

19 Lorsque arriva le temps où Mérab, fille de Saül, devait être donnée à David, elle fut donnée pour femme à Adriel, de Mehola.

20 Mical, fille de Saül, aima David. On en informa Saül, et la chose lui convint.

21 Il se disait: Je la lui donnerai, afin qu'elle soit un piège pour lui, et qu'il tombe sous la main des Philistins. Et Saül dit à David pour la seconde fois: Tu vas aujourd'hui devenir mon gendre.

22 Saül donna cet ordre à ses serviteurs: Parlez en confidence à David, et dites-lui: Voici, le roi est bien disposé pour toi, et tous ses serviteurs t'aiment; sois maintenant le gendre du roi.

23 Les serviteurs de Saül répétèrent ces paroles aux oreilles de David. Et David répondit: Croyez-vous qu'il soit facile de devenir le gendre du roi? Moi, je suis un homme pauvre et de peu d'importance.

24 Les serviteurs de Saül lui rapportèrent ce qu'avait répondu David.

25 Saül dit: Vous parlerez ainsi à David: Le roi ne demande point de dot; mais il désire cent prépuces de Philistins, pour être vengé de ses ennemis. Saül avait le dessein de faire tomber David entre les mains des Philistins. 
26 Les serviteurs de Saül rapportèrent ces paroles à David, et David agréa ce qu'il lui était demandé pour qu'il devînt gendre du roi.

27 Avant le terme fixé, David se leva, partit avec ses gens, et tua deux cents hommes parmi les Philistins; il apporta leurs prépuces, et en livra au roi le nombre complet, afin de devenir gendre du roi. Alors Saül lui donna pour femme Mical, sa fille.

28 Saül vit et comprit que l'Eternel était avec David; et Mical, sa fille, aimait David.

29 Saül craignit de plus en plus David, et il fut toute sa vie son ennemi.

30 Les princes des Philistins faisaient des excursions; et chaque fois qu'ils sortaient, David avait plus de succès que tous les serviteurs de Saül, et son nom devint très célègre. ${ }^{15}$

Il faut l'admettre, même ceux d'entre nous que la grâce du Seigneur a'a pas visités reconnaîtront l'extraordinaire séquence de coincidences qu'une lecture peut faire maintenant se superposer entre les deux séries: selle de la vie de Charles III, celle de David. Nous nous poserons plus tard les questions qui portent sur les conséquences, pour la "théorie" de cette lecture; ou encore: que faut-il supposer pour qu'une telle lecture soit possible?

Pour le moment, nous devons rester dans le problème de la date. Nous savons, ou du moins, pour accepter notre hypothèse nous devons supposer, que cette trilogie n'a pas été écrite avant janvier 1559, date où Charles devint le gendre de Henri II. D'autre part, la copieuse Epistre dédicatoire nous donne la date de $1563^{16}$ comme date de rédaction, : innée où il envoie son oeuvre achevée à un certain Le Brun que Lebègue ie peut pas identifier. Dans cette Epistre pourtant se trouvent suffisamnent d'indices pour que nous puissions situer encore plus précisément a composition.

Encore un effort: la critique de la Renaissance est une longue patience. '̇xemple: si Legègue n'a pas identifié Brun, c'est qu'il est pour lui au :ervice d'une certaine comtesse d'Acier, ${ }^{17}$ alors qu'il s'agit d'Assier(Lot); ('ette comtesse est d'autre part comtesse du Raing chez Monluc, ${ }^{18}$ alors que pour nous elle est comtesse du Rhin. Elle est mariée depuis 1550 à Jean-Philippe de Salm, comte palatin du Rhin; voisin du duché de Lorraine, qui est ce même personnage qui mit au service de la catholique couronne de France les troupes allemandes levées par et pour les protestants en juillet $1562 .{ }^{19}$

Or donc Le Brun est à Assier en 1563. L'Epistre nous dit aussi que lorsqu'il partit, Le Brun espérait un prompt retour, mais qu'il n'en a rien été; qu'il est parti pour prêter main-forte à la Comtesse, au moment de troubles à Montauban et dans le Lot. Or les mémoires de Monluc nous apprennent que ces troubles se situent fin 1561 , début $1562^{20}$; ce que 
confirment aussi les vers 15 sq. de l'Epistre. Des Masures a dû fuir au début de cette même année. Le Brun semble ne rien connaître de ces événements, ou du moins Des Masures n'a aucune raison de penser qu'il les connaît. L'Epistre incite aussi à penser que Le Brun n'a jamais vu les tragédies. ${ }^{21}$ Ce qui nous situerait la composition entre la fin 1561 et 1563.

Reste cependant la question de la représentation. Tout ce que nous savons du théâtre écrit au XVIème siècle nous interdit de penser que l'on pouvait écrire pour la publication seule. Les textes sont bien faits pour être représentés, et probablement une seule fois, ${ }^{22}$ à l'occasion d'un événement important. Les mystères font traditionnellement partie des fêtes, celles du saint patron, action de grâces. Certains sont utilisés presque comme voeu propitiatoire, et sont offerts au saint éponyme pour prévenir une épidémie de peste par exemple. ${ }^{23}$ Mais l'occasion la plus commune était celle de l'entrée dans une ville ou sur un territoire d'un personnage de marque, ce qui justifiait le déploiement important de ressources que la mise en scène de pareils spectacles exigeait.

Il faudrait donc essayer de retrouver des devis, commandes, etc., relatifs à une mise en scène quelconque. Nous en avons effectivement quelques traces de préparatifs d'entrée dans les Archives de Meurthe-etMoselle, en janvier 1558 , et aussi en $1559 .{ }^{24}$ Or une entrée solennelle, que Charles III aurait dû faire, s'accompagnait traditionnellement, de la reconnaissance de certains droits. Dans le cas de Nancy, il s'agissait de reconnaître les droits de la noblesse de Lorraine. Or Charles III remet par deux fois son entrée, précisément parce qu'il ne veut pas reconnaître ces droits, et, de plus, probablement, parce que la situation politicereligieuse est trop fluide: on se demande après la mort de François II $^{25}$ de quel côté va pencher le jeune duc; ${ }^{26}$ on voit aussi qu'il rentre lorsque les deux camps sont irrémédiablement en guerre et qu'il a décidé de poursuivre les huguenots en Lorraine. Il ne peut être question d'une représentation pour Des Masures en mai 1562. On avait prévu une entrée en 1558, qui fut décommandée.

L'hypothèse de la rédaction devient alors: Des Masures prévoit une représentation théâtrale pour l'entrée de Charles III et de sa nouvelle épouse. La conjecture, ici, est faite à peu près à coup sûr, et sur la probabilité de la représentation théâtrale, et sur l'identité de celui à qui serait commandée la pièce puisque Des Masures est l'homme de lettres officiel du duché. A cette occasion heureuse correspond effectivement très bien le David triomphant qui reprend le sujet d'une entrée triomphale, de l'amitié du fils du roi et de David, du double mariage des filles du roi, dont la cadette a épousé David. Rencontre extraordinaire en effet, où le jeune duc aurait vu sa propre vie représentée, où l'entrée réelle se double de l'entrée sur scène.

Le David combattant et le David fugitif servent à établir le sens pro- 
testant de David triomphant, et lui seraient postérieurs, établissant David comme incarnation de l'élu de Dieu (l'exemplaire) et Saül (Henri II de France) comme exemplaire de l'abandonné de Dieu.

Le problème initial de l'efficacité politique d'une pièce de théâtre au XVIe soulève toutes les questions du retentissement de l'idéologique sur les autres instances - ici le politique - et même, parce que nous sommes au XVIème siècle, de la place de l'idéologique dans la formation sociale, et même, de l'existence d'une instance idéologique dans la formation sociale en question.

Reprenons. Si nous examinons l'interprétation classique, elle nous apparaît reposer sur l'identification de David à l'auteur, selon un mode que nous appellerons rapidement romantique, c'est-à-dire au fond, romantique-tel-que-le-voit-la théorie-critique de Lebègue. Littéralement, celui-ci ne voit pas les autres identifications signalées pourtant clairement dans L'Epistre qui est le texte sur lequel il s'appuie pour assurer son identification:

1. Le Brun v.31

2. Des Masures (ce qui est vu par Lebègue) v. 35

3. "nostre bon duc lorrain" 99 sq.

Quel est don cet aveuglement? Doit on la situer dans l'infirmité du critique, comme le font immanquablement les tenants de ce que Lefort appelle le réalisme? Non; c'est tout simplement que dans la théorie de Lebègue certains éléments du texte ou de l'histoire n'apparaîtront pas comme faits; ils seront rejetés du côté de l'extérieur, du non-signifiant; du secondaire, de l'insignifiant. Ainsi c'est tout un de désigner par le découpage de la littérature "l'intention moralisante" et "la propagande religieuse" comme hors-champ et de ne pas voir accéder au statut de faits la paix de Cateau-Cambrésis et le double mariage.

Pourtant il est tout aussi "réaliste" de croire qu'en interrogeant l'histoire seule, celle-ci, ventriloque, produira pour nous les faits qui nous permettront de changer de théorie. Le mouvement est bien ici de réintégrer les donnés "secondaires" et de voir ce que donne ce déplacement de la question; celle-ci se pose effectivement ailleurs, non plus dans le littéraire, ni dans le moral, ni dans le religieux, mais dans le politique; ce qui ne veut pas dire que la question n'est plus morale, ni religieuse ni littéraire: elle l'est autrement.

Politiquement, quelles sont alors ces pièces de théâtre offertes à un souverain qui n'a pas encore choisi sa voie, qui représentent l'Ecriture sainte pour répéter la vie du duc, et qu'est-ce que cette répétition qui a eu lieu de toute éternité déjà? ${ }^{27}$ 


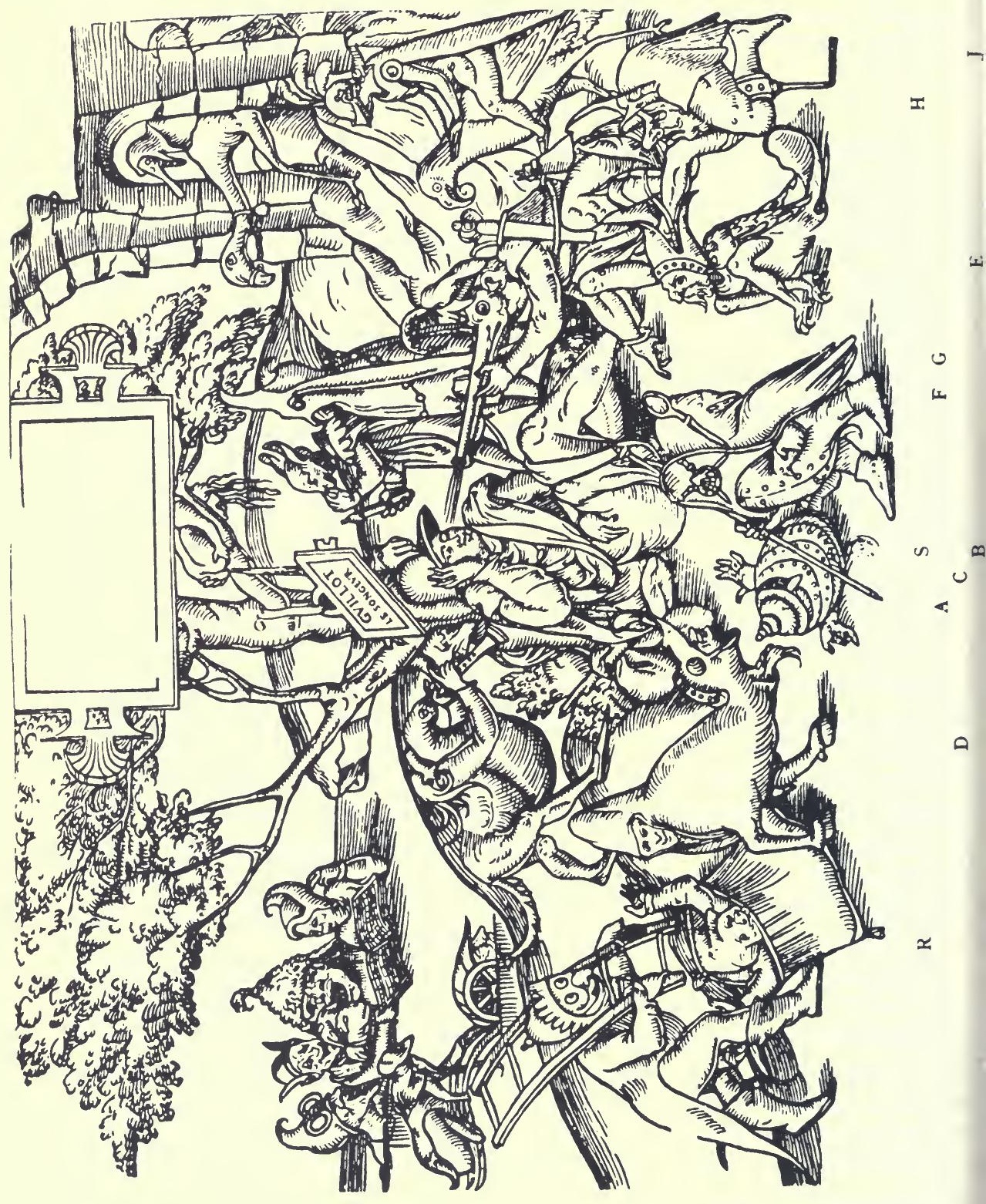

Tiré de: Théodore de Bèze, Correspondance, éditée par H. Albert et al. (Genève: Droz, 1963). 


\section{GRAVURE DE GUILI.OT LE SONGEUR}

(Bil). nationale de Paris, Estampes, coll. Hennin, t. IV, p. 25, seul exemplaire connu; a vant la lettre.)

Briève exposition de la painlure ensuyvantc que a esté sémée en France, concernant le présent estat de la Court [peu après la inort de François II]

(Texte figurant dans les Mém. de Condé, t. II, p. 655 s.)

A. L'arbre signifie le Royaulme de France.

B. Guillot le songeux, est le Roy de Navarre ou Duc de Vendosme, qui s'appuye contre iceluy, \& dort comme nonchallant, \& qui ne se soulcie pas grandement de l'Estat du Gouvernement \& de la présente occasion.

C. l:Admiral de France, qui pour le réveiller, le tire par le chappeau.

D. Le Cardinal de Chastillon qui luy souffle aux aureillez de debvoir mettre les mains audict Gouvernement.

E. Le Connestable qui par toutes sortes d'armes \& guerres, met paine de le réveiller

F. Le Prince de Condé son frere, qui luy porte une chandelle allumée, pour luy monstrer tant plus clairement comme l'on l'avoit traíné par le né, de sorte qu'il avoit desja quasi perdu la teste.

G. Le Cardinal de Bourbon qui va masqué sans se déclairer pour nulle des Partyes, pour veoir ce qu'en sera.

H. Le Visdame de Chartres prisonnier, lequel se monstre en partie dehors \& en partie en dedans la porte, pour ce que aulcungs disent qu'il est mort en prison, \& aultres en liberté.

J. Le Baron de Marigny [Maligny] qui practica l'esmotion \& révolte d'Amboise, \& voyant que la chose ne procédoit pas comme il l'avoit pourgecté, il s'en désenveloppa, \& parla pour les aultres; \& pourtant chevauche-il espéronné sur ung papagay.

K. Monsieur De Guyse, lequel estant Grand-Maistre \& Grand-Chasseur de France, se trouve présentement sans Chief, \& va errant comme ung pellerin, cherchantz son passe-temps par la vollerie \& porte la lonche [louche] pour s'estre entremis à toutz affaires, jusques à entendre les minuteries [minuties] de la Cuysine.

L. L'Evesque d'Amiens qui le' suyt toutzjours.

M. Le Maresclial de Brisac, gouteux \& grand cracheur.

N. Le Duc de Lorraine, duquel comme jeusne, \& qui n'est encores du tout sorty de l'escaille, l'on ne sçait encores de qui cousté il se tournera.

O. Le Royne-Mere, ayant deux visaiges, tournant l'ung vers le Roy de Navarre, comme Royne, \& riant pour luy complaire; \& l'aultre contre le Cardinal de Lorraine, comme une bonne vieille \& simple chamberiere, obéissante à ses commandementz.

P. Le feu Roy François le deuxiéme, lequel le Cardinal tenoit en sa bourche [bourse], le laissant quelquesfois mettre dehors la teste ou les mains, pour prendre ayr.

Q. Le Cardinal de Lorraine qui estoit luy-mesme Roy ; \& pour aultant porte-il la Couronne, \& une eschelle sur son col, pour avoir avallé chés luy tout ce qu'il a peu ; \& pource que le bruict fust qu'il faisoit l'amour a la jeusne Royne, l'on l'ha mis en telle disposition.

R. Les Ecclésiastiques, lesquelz pour estre mal-fondés \& sur l'eaive, nonobstant qu'ilz chantent, cryent \& se plaignent grandement, se vont au fond noyantz.

S. C'est que le Roy de Navarre se laisse gouverner \& empescher par Dames et amouretes, de sorte qu'elles en font de luy ce qu'elles veullent. 
Car c'est bien la seule efficacité que l'on puisse espérer au niveau politique. Politiquement, le seul public, c'est le duc: le seul qui puisse être transformé par la représentation de façon à ce que la vérité reprenne le monde. Symétrie du sujet (le représenté) de la représentation et de celui qui la regarde qui ressemble étrangement à celle que révèle Foucault dans son analyse des Ménines.

Reste encore que si l'on admet que le $\operatorname{Sujet}^{28}$ a pas encore eu lieu, pas encore de place, on ne peut guère lui garder un refuge à l'intérieur de ce souverain qui vient de prendre place devant le spectacle pour lui donner occasion et sens. Sens unique, intransportable, à moins qu'on n'évacue la possibilité de transformation du monde qu'implique la présence de la souveraineté devant ce théâtre à ce moment-là et en ce lieu-là. La présentation tragique est la saisie dans l'instant présent de la superposition unique de deux séries guidées de toute éternité par la Providence. Dans cette répétition des événements se lit un Destin chrétien, et la poésie tragique protestante est un peu de même nature que la mantique stoïcienne: montrer les points d'apparition du Destin dans l'histoire humaine. A la limite, il n'y a plus d'événement: tout est annonce ou rappel. Ainsi David annonce Charles III, et Charles III répète David: tout est déjà représentation, double, rappel, approximation, révélation. Pour nous, plus tard, et comme le dira Des Masures lui-même dans son Epistre, à qui lira (le petit Masurim son fils) ou "quiconque aura désir,"

\section{quiconque par loisir \\ Lire ou représenter ces vers aura désir ${ }^{29}$}

il ne restera que la leçon morale, l'éducation, la catharsis, la généralité, tout ce qui peut subsister une fois résolue en texte la conjonction unique et vivante du spectacle.

Ce que dit alors la représentation tragique, c'est que l'histoire n'est que re-présentation tragique, que la tragédie se répète et que l'histoire n'est que la répétition de cette tragédie. Ainsi sont éclaircis les deux premiers mots du titre de cette étude, et le dernier, pour ce qui est du rapport du Prince au texte.

Reste encore la place de l'auteur. Si l'histoire marche ainsi du pas égal de la répétition déterminée par la providence divine, quel secours espérer du travail de l'oeuvre, qui d'être littéraire (du moins la disions-nous telle) n'en est pas moins soumise aux mêmes lois que toutes les oeuvres du monde dans l'univers protestant: elles sont la vanité même, pure manifestation, au mieux, de l'élection de l'auteur par la grâce gratuite; au pire, travail incessant de Satan? Cette vanité de l'oeuvre a bien sür pu être reprise dans l'interprétation "romantique" que je signalais tout à l'heure: sans efficace propre, on l'a reversée au compte du narcissisme, du chant 
désespéré sur soi, que les Psaumes si souvent repris dans les tragédies protestantes manifesteraient aussi: lyrisme, épanchement.

Mais qui ne voit que ceci est interprétation, et que ce "lyrisme" est une sorte d'effet de surface de la théorie qui voit une oeuvre et un auteur. A faire disparaître pour un moment celle-là et celui-ci, on pourrait envisager de relire les vers suivants de l'Epistre:

Que pleust au Souverain

Qu'en cette école instruit nostre bon Duc Lorrain

Conformast à David entièrement sa vie,

Et que son âme à Dieu par foi jointe et ravie

Meditast jour et nuict, ainsi que ce bon Roy,

Du Seigneur souverain la souveraine Loy. ...

(v. 99-104)

D'où je tire: (1) la souveraineté n'est pas dans le Prince. Nous ne pouvons non plus le mettre en position de sujet donneur du sens que le "public" dont dépendait l'interprétation de Lebêgue: (2) le sens ne sera pas donné par l'interprétation; il ne peut être compris par la raison sans la grâce. Le sens ne viendra que dans la perte de soi, dans l'union à Dieu, au-delà du rationnel et de tout discours. Il ne s'agit pas de comprendre, il s'agit de répéter: certains philosophes n'ont fait qu'interpréter le monde, il s'agit maintenant de le répéter. Ainsi l'Histoire qu'il faut bien nommer Providence est ce continu tragique inaccessible dont la tragédie est la scansion et la manifestation. Je dirais volontiers, en jouant sur l'anglicisme et sur le sens linguistique: le spectacle est la performance et non la représentation.

La tragédie est l'histoire du Prince, qui se trouve ainsi agi et agent.

Reste dans le titre le mot service.

Il est là pour indiquer la relation de l'auteur - du répétiteur? - au Prince. Que penser de cette instruction et de cette escole ${ }^{30}$ Il ne peut s'agir encore une fois de perfectibilité, de dépassement de soi dans la prise de conscience et autres saisies de soi humanistes. Tout est dans la coïncidence miraculeuse, dans la fulguration de la rencontre de la Loi, du Prince et du texte: pas de contrainte par le rationnel ou par le pragmatique. Toute l'intériorité que nous pourons supposer au Prince ne va qu'à acquiescer volontairement à la vie qui lui est proposée: "se conformer" à "l'exemplaire," à la "preuve apparente." ${ }^{31}$ Le texte est simplement proposé, proféré, offert au risque de se perdre si Dieu n'a pas insufflé la grâce dans l'âme du Prince. Risque mortel de la performance qui désormais ne se survivrait, comme nous l'avons vu, que dans l'écrit.

Ici encore, pas d'oeuvre: un acte offert au Prince comme le service l'est au moment de l'allégeance. Comme le supplément de grâce est 
donné au Prince, comme l'épée est donnée au chevalier, ainsi le sens vient au texte d'en haut, en une suite descendante de dons qui se lisent à chaque niveau par l'inscription du manque.

$\mathrm{Si}$ j'ai bien fait mon travail, peut-être pourrez-vous comprendre que chacun des mots du titre n'est que le miroir et le redoublement de chacun des autres. Que le titre est tautologique; pas tout à fait pourtant: derrière cette confusion que je viens de produire flotte encore comme une rémanence la distinction de chacun des termes, produisant ainsi la troisième dimension d'un espace où précisément la confusion est mouvement.

Queen's University

\section{Notes}

1 David combattant, David triomphant, David fugitif (1563? v.p. 13) éditées par Ch. Comte sous le titre de Tragédies saintes S.T.F.M. 1932

2 Emile Faguet: La tragédie française au XVIe siècle (Paris: N. Welter, 1894).

3 Raymond Lebègue: La tragédie religieuse en France Les débuts (1514-1573) (Paris: Honoré Champion 1929), pp. 327-368.

4 Emile Faguet,op. cit., pp. 119-120.

5 Lebègue, op. cit., p. 367.

6 Lebègue, op. cit., p. 368.

7 Ibid.

8 "Il porte pour cuirasse un blanc rochet rural:

Il tient au poing, pour lance, un baston pastoral:

La targe est sa mallette, et l'armet son chappeau:

Et en lieu d'une armée il conduit un trouppeau."

Des Masures "Prologue" de David combattant, v. 41-44.

9 Lebègue, op. cit., p. 337.

10 V.p. 14.

11 Lebègue, op. cit., p. 345.

12 Lebègue, op. cit., pp. 349-350.

13 Lebègue, op. cit., p. 364.

14 Voir R. Chartier et al., L'éducation en France du XVIe au XVIIIe s. (Paris: Société d'Edition de l'enseignement supérieur, 1976).

15 I Samuel 18 - trad. Louis Segond (Genève: Maison de la Bible).

16 V. 151 de l'Epistre. Cf. Lebègue, op. cit., p. 328.

17 Lebègue, op. cit., note 3, p. 340.

18 Blaise de Monluc, Commentaires, Bibliothèque de la Pléiade, p. 489.

19 Voir note 4 p. 101 au Tome IV de la Correspondance de Th. de Bèze, recueillie par H. Aubert, Travaux d'Humanisme et Renaissance LXXIV (Genève: Droz, 1965).

20 Massacre de hugenots le 16 nov.: v. Monluc note 7, p. 479, op. cit. Montauban: siège mars 1562.

21 (Epistre) vv. 63-65.

22 Eas (tragoedias) enim, ut consuetudini scholae satisfeceret quae per annos singulos singulas poscebat Fabulas, conscripserat. 


\section{Renaissance et Réforme / 161}

G. Buchanan Vita $a b$ eo scripta, 1539. In Lanson, "Etudes sur les origines de la tragédie classique," R.H.L.F. (1903), p. 183, note 1.

23 Jacques Chocheyras, Le théâtre religieux en Dauphiné du Moyen-âge au XVIIIème siècle (Genève: Droz, 1975), p. 53.

24 Documents B1118 et B1120 des Archives du Meurthe-et-Moselle.

255 décembre 1560.

26 Voir N. dans la gravure qui se trouve à la page 156 de cet article, pp. 78 et 79 de Correspondance de Théodore de Bèze, Tome III (1559-1561), Travaux d'Humanisme et Renaissance LXI (Genève: Droz, 1963).

27 Ainsi M. Philone - sur l'identité duquel on s'interroge encore - écrit un Josias (1566), dont le sous-titre est "vrai miroir des choses advenues de notre temps" - v. Lebègue, op. cit., pp. 323-326.

28 Celui de la philosophie classique.

29 Vv. 28-29.

30 [Epistre] v. 100; v. plus haut.

31 "Or David (si à nul peuvent ces carmes plaire)

En fait preuve apparente, et en est l'exemplaire"

(Epistre) vv. 117-118. 